\title{
Jack 3/5 State from Two-Body Interaction
}

\author{
B. Kuśmierz ${ }^{a}$, Y.-H. WU ${ }^{b}$ AND A. WóJS ${ }^{a}$ \\ ${ }^{a}$ Department of Theoretical Physics, Wrocław University of Technology, \\ Wybrzeże Wyspiańskiego 27, 50-370 Wrocław, Poland \\ ${ }^{b}$ Max-Planck-Institute of Quantum Optics, Hans-Kopfermann-Str. 1, 85748 Garching, Germany
}

We investigate the Read-Rezayi parafermion state of correlated electrons at the fractional Landau level filling $\nu=3 / 5$. It is a Jack polynomial generated by contact four-body repulsion. We show by exact diagonalization that it is also emerges from a suitable short-range two-body interaction. We find that it closely matches Coulomb ground state in the second Landau level of non-relativistic fermions, and thus possibly describes the $\nu=13 / 5$ (and, by conjugation, $\nu=12 / 5$ ) fractional quantum Hall effect in GaAs.

DOI: 10.12693/APhysPolA.129.A-73

PACS: 73.43.-f

\section{Introduction}

Fractional quantum Hall effect (FQHE) [1] reveals spontaneous emergence of a series of correlated, nondegenerate, gapped quantum fluid ground states in a system of essentially two-dimensional (2D) electrons with the Coulomb interaction filling particular fractions $\nu$ of an isolated, massively degerate Landau level (LL) formed in a high magnetic field. Since orbitals of the lowest LL are essentially monomials (in the complex 2D coordinate $x$ ), the many-electron wave-functions defining universality classes for different FQH states are seeked among complex antisymmetric polynomials. (This also applies to FQHE in higher LLs, via mapping onto the lowest LL.)

\section{Theory}

Virtually all known FQH states are explained by the composite fermion (CF) theory [2] which postulates binding by electrons an even number $(2 p)$ of vortices of the many-body wave function. Most invoke one kind of essentially free CFs filling an integral number $n$ of effective CF LLs $(\Lambda \mathrm{Ls})$; this is the Jain series of fractions: $\nu=n /(2 p n \pm 1)$, equating FQHE with integral $\mathrm{QHE}$ of CFs. Others depend on $\mathrm{CF}-\mathrm{CF}$ interaction and their description involves extensions such as $\mathrm{CF}$ pairing/condensation [3] or additional CF degrees of freedom ("partitions") [4]. Among the latter is the "parafermion" $\nu=k /(k+2)$ series of states [5] generated as unique zeroenergy ground states of contact $(k+1)$-body repulsions, exemplifying the Jack polynomials [6].

The Jack polynomials $J_{\lambda}^{\alpha}$ are indexed by integer partitions $\lambda$ and real numbers $\alpha$. They are eigenstates of the following Laplace-Beltrami Hamiltonian defined in the space of symmetric polynomials

$$
\begin{aligned}
& H_{\mathrm{LB}}(\alpha)=\alpha \sum_{i}\left(x_{i} \partial_{i}\right)\left(x_{i} \partial_{i}\right) \\
& \quad+\sum_{i<j}\left(x_{i}+x_{j}\right)\left(x_{i}-x_{j}\right)^{-1}\left(x_{i} \partial_{i}-x_{j} \partial_{j}\right) .
\end{aligned}
$$

An explicit recursion construction exists for symmetric Jack polynomials [7]; fermionic Jacks have an additional antisymmetrizing Vandermonde factor.
The Jacks relevant for FQHE must yield uniform wave functions; in the Haldane spherical [8] geometry this means no total angular momentum $L=0$. This sets the value of $\alpha$, while partition $\lambda$ determines the filling factor $\nu[9]$.

\section{Results}

Let us consider a particular Jack: Read-Rezayi (RR) $k=3$ parafermion state [5], expected to form in the first excited $(n=1)$ LL of massive spinless electrons and thus a plausible candidate for the $\nu=13 / 5 \mathrm{FQHE}$ in GaAs.

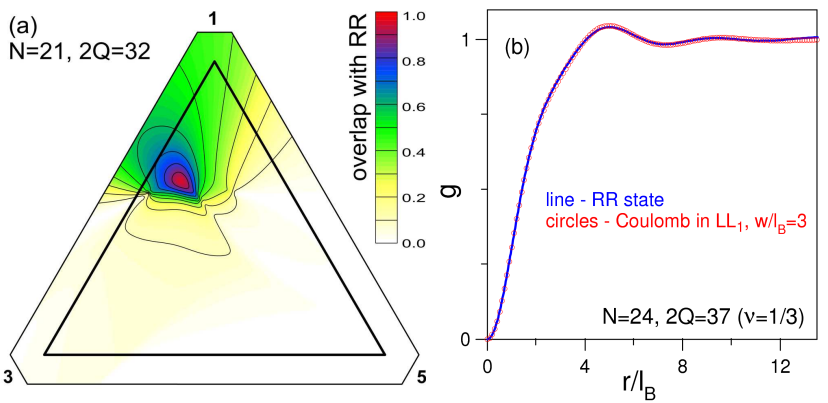

Fig. 1. (a) Contour map of overlaps of the RR state with ground states of two-body pseudopotentials $\left(V_{1}, V_{3}, V_{5}\right)$, calculated on a sphere for $N=21$ particles. Corners of the inner triangle correspond to one positive pseudopotential $V_{m}$ ( $m$ as indicated) and all others vanishing; the inside to $V_{m=1,3,5}>0$ and $V_{m>5}=0$ [10]. (b) Pair correlation functions $g(r)$ of RR state and Coulomb ground state in the $n=1$ LL (layer width $w / l_{B}=3 ; l_{B}$ is the magnetic length), calculated for $N=24$.

First, we checked if it can be accurately generated by a suitable short-range two-body interaction. We diagonalized two-body interactions with arbitrary three leading pseudopotentials $V_{1}+V_{3}+V_{5}=1\left(V_{m}\right.$ defined as dependence of pair energy $V$ on relative angular momentum $m$ [8]). In Fig. 1a we show the map of overlaps of the lowest $L=0$ eigenstate of $\left(V_{1}, V_{3}, V_{5}\right)$ with the RR state, obtained for a fairly large system of $N=21$ particles 
on a sphere (results for smaller systems are similar). Indeed, for $V_{1}: V_{3}: V_{5} \approx 6: 3: 1$ the overlap reaches $97 \%$, which demonstrates that a suitable two-body interaction can simulate a higher-order interaction.

Second, from similar maps we have also determined optimum model interactions $\left(V_{1}, V_{3}, V_{5}\right)$ which simulate Coulomb ground states in different LLs. We found that only the $n=1 \mathrm{LL}$ in GaAs (especially in wider quasi-2D layers) is simulated by nearly the same model as the RR state.

Finally, we have directly compared the RR and Coulomb states; the overlaps are listed in Table I; the pair correlation functions are drawn in Fig. 1b. Indeed, the $\nu=13 / 5$ FQHE in GaAs appears a manifestation of the RR state, described by a Jack wave function. By particle-hole conjugation, the (also observed) $\nu=12 / 5$ FQHE state is the corresponding "anti-Jack".

\section{TABLE I}

Overlaps of the RR state with different Coulomb $L=0$ ground states on a sphere. Columns: electron number $N$, magnetic flux $2 Q$, dimension of the diagonalized $N$-body subspace with $L_{z}=0$, and the overlaps with Coulomb states in the $n=0$ and 1 LLs in GaAs $\left(\mathrm{LL}_{n}\right)$ and in the $n=1$ and 2 LLs in graphene $\left(\mathrm{G}-\mathrm{LL}_{n}\right)$. Layer width is zero except for $\mathrm{LL}_{1}^{\text {wide }}$ corresponding to 3 magnetic lengths.

\begin{tabular}{c|c|c|c|c|c|c|c}
\hline \hline$N$ & $2 Q$ & Dim & $\mathrm{LL}_{0}$ & $\mathrm{LL}_{1}$ & $\mathrm{LL}_{1}^{\text {wide }}$ & $\mathrm{G}_{-L_{1}}$ & $\mathrm{G}_{-} \mathrm{LL}_{2}$ \\
\hline 18 & 27 & $2 \times 10^{5}$ & 0.5399 & 0.9369 & 0.8995 & 0.5458 & 0.3584 \\
21 & 32 & $5 \times 10^{6}$ & 0.5689 & 0.8990 & 0.9316 & 0.5714 & 0.1332 \\
24 & 37 & $1 \times 10^{8}$ & 0.3442 & 0.8100 & 0.8792 & 0.3468 & 0.1408
\end{tabular}

\section{Acknowledgments}

We acknowledge helpful discussions with J.K. Jain and support from the following grants: Polish NCN 2014/14/A/ST3/00654 (BK, AW), EU Marie Curie PCIG09-GA-2011-294186 (AW), EU SIQS (YHW). Computations were performed at WCSS and CYFRONET, both parts of PL-GRID.

\section{References}

[1] D.C. Tsui, H.L. Störmer, A.C. Gossard, Phys. Rev. Lett. 48, 1559 (1982).

[2] J.K. Jain, Phys. Rev. Lett. 63, 199 (1989).

[3] G. Moore, N. Read, Nucl. Phys. B 360, 362 (1991).

[4] G.J. Sreejith, C. Tőke, A. Wójs, J.K. Jain, Phys. Rev. Lett. 107, 086806 (2011); G.J. Sreejith, A. Wójs, J.K. Jain, Phys. Rev. Lett. 107, 136802 (2011); G.J. Sreejith, Y.-H. Wu, A. Wójs, J.K. Jain, Phys. Rev. B 87, 245125 (2013).

[5] N. Read, E. Rezayi, Phys. Rev. B 59, 8084 (1999).

[6] I.G. Macdonald, Symmetric Functions and Hall Polynomials, Oxford University Press, New York, 1995; B. Kuśmierz, Y.-H. Wu, A. Wójs, Acta Phys. Pol. A 126, 1134 (2014).

[7] L. Lapointe, A. Lascoux, J. Morse, Elec. J. Combin. 7, N1 (2000).

[8] F.D.M. Haldane, Phys. Rev. Lett. 51, 605 (1983).

[9] B.A. Bernevig, F.D.M. Haldane, Phys. Rev. B 77, 184502 (2008); Phys. Rev. Lett. 100, 246802 (2008); ibid. 102, 066802 (2009).

[10] A. Wójs, Phys. Rev. B 80, 041104(R) (2009). 